\title{
Tumor sólido pseudopapilar del páncreas
}

\author{
Luis Tinoco-Téllez, ${ }^{1}$ Ernesto Marín y Santillán, ${ }^{2}$ \\ Mario Murguía-Pérez, ${ }^{3}$ Luis Gerardo Domínguez-Carrillo ${ }^{4}$
}

\section{Resumen}

Antecedentes: El tumor sólido pseudopapilar del páncreas es un tumor epitelial de baja malignidad; constituye 1 a $2 \%$ de todas las neoplasias del páncreas y se presenta en mujeres jóvenes. Caso clínico: Masculino de 23 años de edad con síntomas de saciedad temprana, distensión abdominal postprandial y cólicos ocasionales de seis meses de evolución. A la exploración: masa abdominal localizada en el mesogastrio, confirmada con ultrasonido y tomografía, siendo dependiente del páncreas. Fue sometido a resección; se llegó al diagnóstico de tumor sólido pseudopapilar. Presentó como complicación diabetes mellitus, en control por endocrinología y sin otras complicaciones. Conclusiones: El tumor sólido pseudopapilar de páncreas es una neoplasia rara, siendo extraordinaria en hombres. El presente caso es el sexto reportado en México, y el primero reportado en hombres en nuestro país. Las manifestaciones clínicas incluyen dolor abdominal, sensación de plenitud o saciedad temprana, masa abdominal, náusea y vómito; no obstante, el paciente puede estar asintomático. La tomografía axial computarizada es el método diagnóstico de elección, revelando una masa heterogénea grande encapsulada. El diagnóstico se realiza mediante biopsia; la cirugía tiene un nivel de curación excelente, con supervivencia del $97 \%$ a los cinco años.

Palabras clave: Tumor sólido pseudopapilar del páncreas.

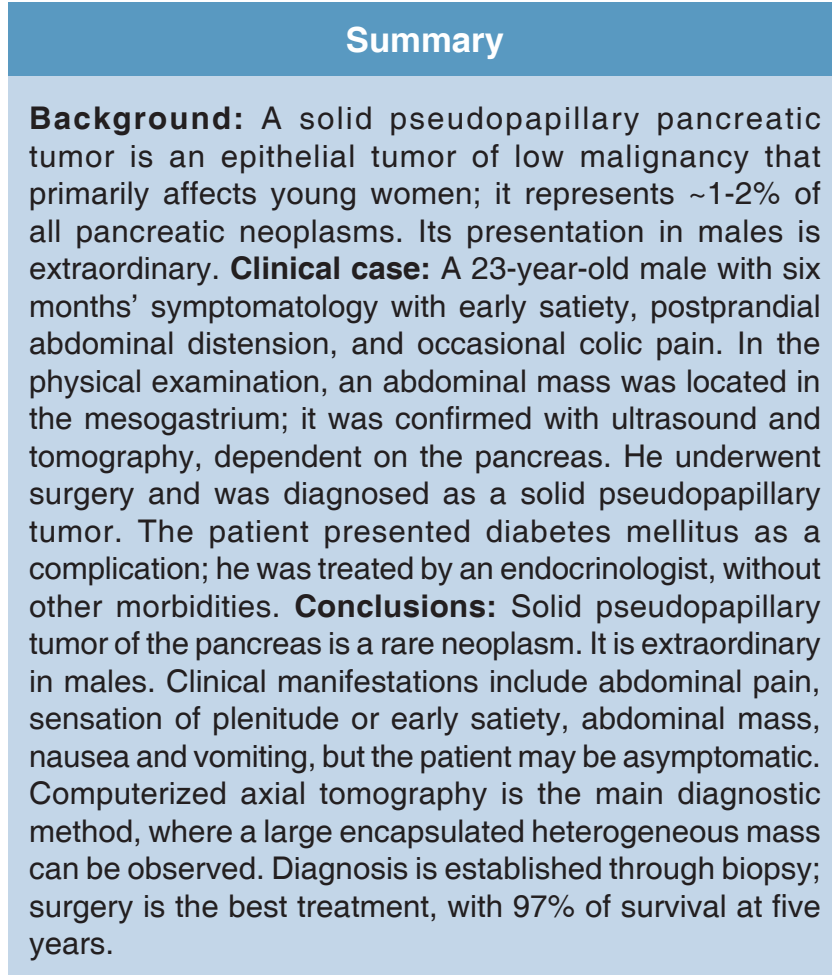

Key words: Solid pseudopapillary pancreatic tumor.

\footnotetext{
${ }^{1}$ Cirujano oncólogo adscrito al Servicio de Oncología Quirúrgica del Hospital Ángeles León.

${ }^{2}$ Cirujano general. Jefe de la División de Cirugía del Hospital Ángeles León.

3 Jefe del Laboratorio de Anatomía Patológica Oncológica y Citología Exfoliativa DIME, IUMAE Núm. 1, IMSS, León, Guanajuato.

${ }^{4}$ Especialista en Medicina de Rehabilitación. Profesor de la Facultad de Medicina de León, Universidad de Guanajuato.
}

Correspondencia:

Dr. Luis Tinoco-Téllez

Correo electrónico: drtinoco_oncología@yahoo.com.mx

Aceptado: 20-10-2015.

Este artículo puede ser consultado en versión completa en http://www.medigraphic.com/actamedica 


\section{INTRODUCCIÓN}

El tumor sólido pseudopapilar del páncreas (TSPP) ${ }^{1}$ es una neoplasia rara de origen epitelial, de etiología desconocida. Fue descrito por Frantz en 1959. Se observa principalmente en mujeres jóvenes no caucásicas; en hombres es extraordinario. La Organización Mundial de la Salud le asignó su nombre actual en 1966. Se le clasifica dentro del grupo de tumores exocrinos del páncreas con potencial maligno incierto. Clínicamente, el paciente portador de TSPP permanece asintomático hasta en 38\% de los casos, o con manifestación de dolor abdominal en $62 \%$, sin características particulares. Al presentarse un individuo masculino en el que se llegó a dicho diagnóstico, que correspondió al sexto caso descrito en México, el primero en hombres en nuestro país, y el primero reportado en la ciudad de León, Guanajuato, nos dimos a la tarea de presentar el proceso motivo del presente trabajo.

\section{CASO CLÍNICO}

Paciente masculino de 23 años de edad con antecedentes de tabaquismo, alcoholismo y toxicomanías positivos, contaba con 55 tatuajes, sin otros antecedentes de importancia. Inició su padecimiento con seis meses de evolución, con distensión abdominal progresiva, exacerbada con la ingesta de sólidos, acompañada de saciedad temprana. Tenía dolor abdominal moderado tipo cólico localizado en el epi- y mesogastrio, sin pérdida de peso ni otros síntomas de relevancia. A la exploración física: sujeto con peristalsis presente en abdomen moderadamente distendido, blando, no doloroso, con una masa palpable en el mesogastrio fija a planos profundos, con dimensiones de $10 \times 12$ centímetros, no dolorosa y sin soplos; el resto de exploración fue normal. Se solicitó ultrasonido, donde se identificó una masa sólida heterogénea sobre el territorio pancreático. Se efectuó tomografía axial computarizada con doble medio de contraste para valorar la extensión de la lesión, y reportó la presencia de un tumor que medía $110 \times 128 \times 97 \mathrm{~mm}$, que mostraba bordes definidos por cápsula, con componente predominante sólido y heterogéneo a expensas de zonas hiperdensas; en las porciones sólidas se observaba un reforzamiento significativo. Se localizaba por delante de la vena esplénica y en íntimo contacto con la porta, sin que existiera una pérdida de interfase entre ambas estructuras en la topografía del cuerpo y cola del páncreas. No se veían los componentes habituales del mismo en su región dorsal, ya que sólo era evidente la cabeza del páncreas a nivel del cuello, justo anterior a la vena porta. El cuello del páncreas se continuaba con la lesión, que comprimía la cavidad gástrica. Se podía apreciar un aumento del diámetro de la vena porta; no se encontraron lesiones en otro sitio ni datos de metástasis o tumores regionales (Figuras 1 y 2). Con los marcadores an- tígeno carcinoembrionario, $\alpha$-fetoproteína, gonadotropina coriónica y antígeno CA-19-9 dentro de los límites normales, y el resto de los paraclínicos normales, excepto DHL elevada $(1,243 \mu / L)$, se realizó una celiotomía exploradora con abordaje a través de la línea media supraumbilical. Se encontró un tumor sólido de aproximadamente 14 centímetros de diámetro localizado en el cuerpo del páncreas, a la izquierda

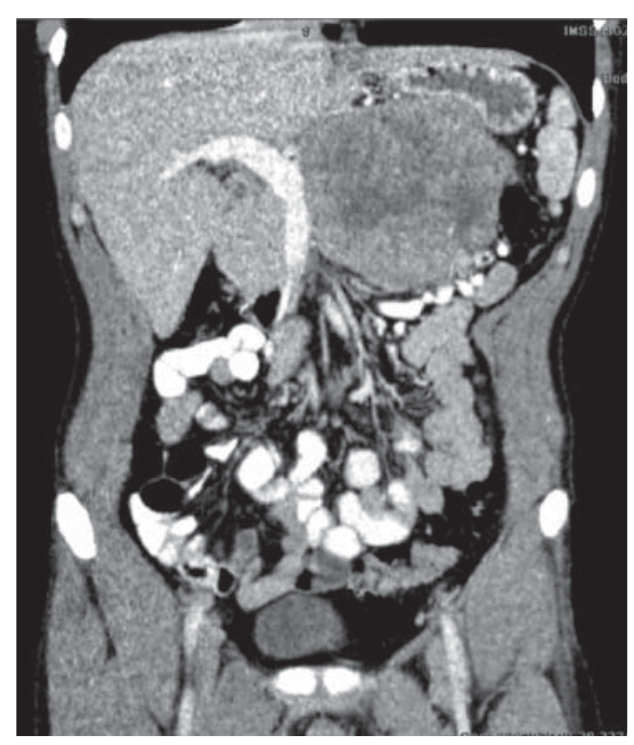

Figura 1. Tomografía axial computarizada en corte coronal de abdomen con doble contraste, en la que se observa una masa de $14 \times 14$ centímetros, sólida, hiperdensa, encapsulada, dependiente del páncreas, que comprime la cavidad gástrica y produce dilatación de la vena porta.

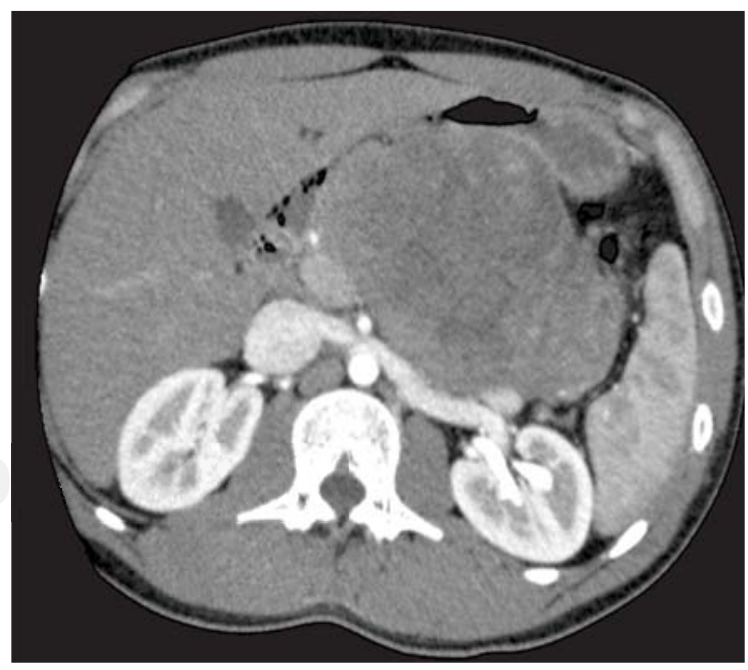

Figura 2. Tomografía axial computarizada en corte axial de abdomen que muestra una lesión tumoral encapsulada y densidad heterogénea dependiente del páncreas. 
de la arteria mesentérica superior; se decidió pancreatectomía distal con esplenectomía, con ligadura de vasos cortos y arteria esplénica a nivel del sitio de resección del tumor. El enfermo se dio de alta al séptimo día postoperatorio.

El estudio histopatológico mostró macroscópicamente un tumor de $14 \times 14 \times 13$ centímetros, con cápsula color gris blanquecino (Figura 3); al corte se encontraron áreas color café oscuro alternando con áreas microquísticas y aspecto congestivo hemorrágico. Microscópicamente se observaron áreas sólidas con células dispuestas alrededor de un estro-

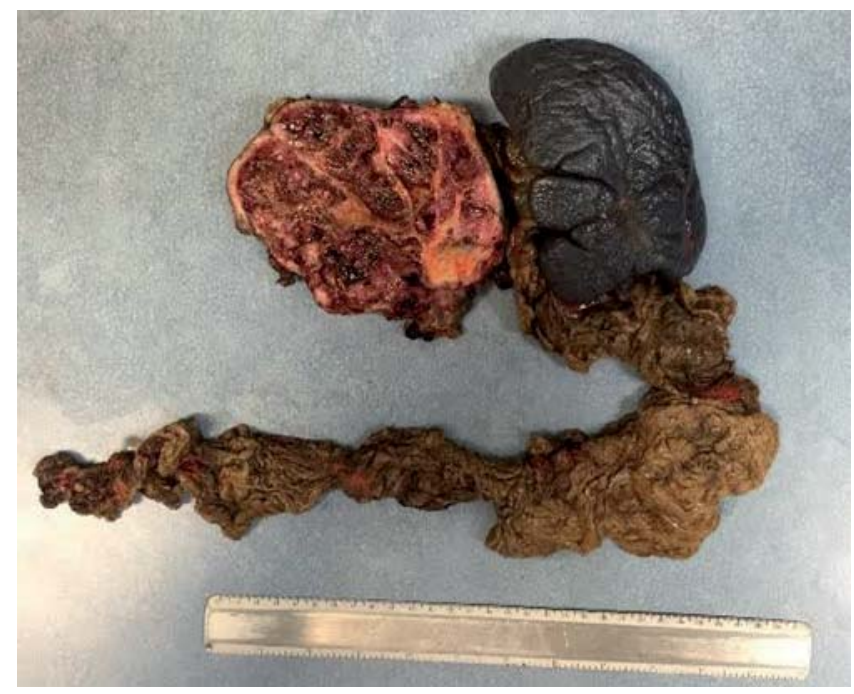

Figura 3. Imagen macroscópica de la pieza quirúrgica, demostrando la cápsula tumoral, el páncreas resecado y el bazo, además de una sección del tumor sólido del páncreas, encapsulado y tabicado.

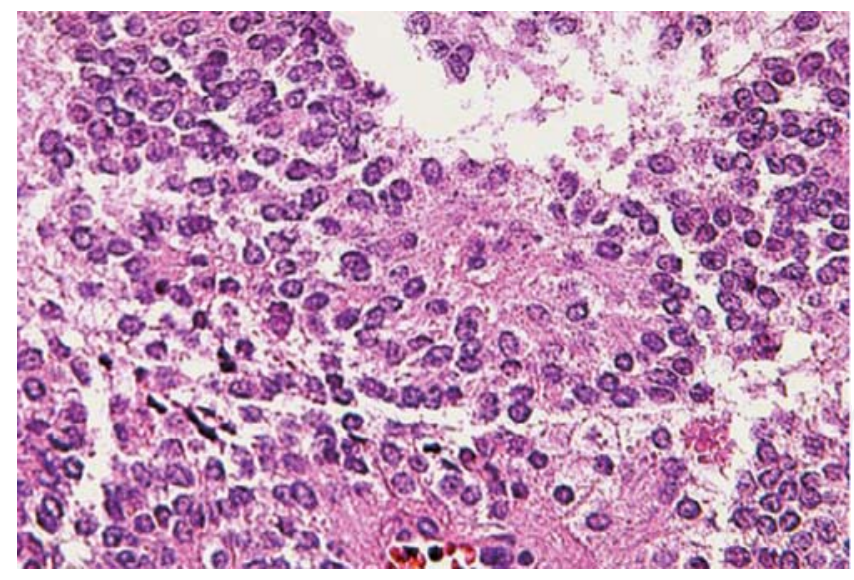

Figura 4. Imagen microscópica del tumor sólido pseudopapilar pancreático, en la que se observan áreas sólidas alternando con cambios degenerativos que producen un patrón pseudopapilar característico de células epiteliales. ma de tejido conectivo fibroso, con capilares congestivos (formaciones pseudopapilares) (Figura 4), además de áreas de necrosis, hemorragia antigua y reciente, con numerosos depósitos de cristales de colesterol; en las células de las pseudopapilas se observaron las hendiduras nucleares características, lo que era compatible con el diagnóstico de TSPP. Desde el punto de vista inmunohistoquímico resultó positivo a vimentina, $\alpha$ - 1 -antitripsina, CD10 y CD56.

Con respecto a la evolución y seguimiento del individuo, permaneció siete días en el servicio; se dio de alta con vía oral y sin complicaciones perioperatorias. Se le citó a los 12 días postoperatorios; no presentó complicaciones de la herida quirúrgica; los paraclínicos de control mostraron glucemia de 140 $\mathrm{mg} / \mathrm{dL}$, por lo que fue canalizado a control por endocrinología, con nueva cita a tres meses para su control por oncología.

\section{DISCUSIÓN}

El TSPP es una neoplasia rara: desde su primera descripción por Frantz, en 1959, hasta 2012 se habían documentado 2,744 casos. En México se habían descrito cinco casos, ${ }^{2}$ siendo el que nos ocupa el número seis reportado en nuestro país y posiblemente, el primero en hombres identificado en México, además de ser el primero descrito en León, Guanajuato y en el Hospital Ángeles León.

El TSPP constituye de 0.2 a $2.7 \%$ de los tumores primarios no endocrinos del páncreas, con predominio en mujeres no caucásicas en más de $90 \%$ de las veces; se presenta especialmente entre la segunda y tercera décadas de vida, ${ }^{1}$ con edad promedio de 28 años. La relación de género es de 1:10 a favor del femenino, lo que significa que se han reportado solamente 335 casos en hombres; ${ }^{3}$ en el género masculino aparece, en promedio, a los 31 años. Su etiología aún está en debate: se ha sugerido su origen en las células del conducto pancreático, las células acinares, las células endocrinas, así como de células pancreáticas pluripotenciales o de tejido extrapancreático (ovárico). Debido a su predominio en mujeres asiáticas y afroamericanas, se argumenta la posible influencia de factores genéticos, pues en $90 \%$ de las muestras obtenidas se encuentran mutaciones en el exón 3 del oncogén de la $\beta$-catenina.

Los estudios inmunohistoquímicos de este tipo de tumor resultan positivos a vimentina, $\alpha-1$-antitripsina, CD10 y CD56 (efectuadas en este caso), enolasa específica de neurona, $\alpha$-1-antiquimiotripsina (no realizadas en este paciente) en más de $90 \%$ de los casos. La presencia de la $\beta$-catenina puede alcanzar $100 \%$ a nivel del citoplasma y $80 \%$ en el núcleo. La cadherina-E se encuentra exclusivamente en el núcleo. El estudio de Gurevich ${ }^{4}$ y colaboradores, realizado en 60 casos con un total de 86 TSPP, indica que este tipo de tumores presenta un inmunofenotipo único, caracterizado por la expresión tanto a nivel nuclear 
como en el citoplasma de claudina; también se ha comprobado que la expresión aberrante de punteado perinuclear de CD99 es una característica única de estos tumores, lo que auxilia en el diagnóstico inmunohistoquímico, logrando la diferenciación con otros tumores pancreáticos.

En la mayoría de los casos de TSPP existen receptores de progesterona en la membrana celular, lo que puede influir en el crecimiento tumoral, pues se ha reportado que éste es mayor durante el embarazo; habitualmente, el índice proliferativo es menor de 1\%. Clínicamente, la sintomatología referida es de dolor abdominal vago localizado en el epi- y mesogastrio en $60 \%$ de los casos (siendo el síntoma más frecuente), ${ }^{3}$ acompañado de sensación de saciedad temprana y distensión abdominal moderada (como en el presente caso); en 30\% de las veces el sujeto se encuentra asintomático y el diagnóstico se establece con una tomografía efectuada por otros motivos, pues la presencia de una masa abdominal, ictericia, náusea, vómito o pérdida de peso es, en general, poco frecuente, por lo que su detección es tardía.

El tumor puede alcanzar 20 centímetros de tamaño (el promedio encontrado en 2,744 casos es de ocho centímetros); crece alrededor de los tejidos y no dentro de los mismos, de ahí la infrecuente obstrucción biliar o pancreática, la cual sólo ha sido reportada en 5\% de los casos, aunque el tumor se localice en la cabeza del páncreas (en el caso específico que presentamos, los valores elevados de DHL sólo correlacionan con la posible congestión hepática demostrada por la distensión de la vena porta detectada en la TAC, ya que el resto de los paraclínicos resultaron normales). Por otra parte, la presencia de abdomen agudo debido a hemoperitoneo por rotura espontánea o sangrado intratumoral es extraordinaria.

El diagnóstico incidental tras la realización de un estudio de gabinete es lo habitual en estos individuos; los exámenes de laboratorios, por lo general, son normales y el diagnóstico del tumor sólido pseudopapilar de páncreas se realiza con estudios de ultrasonido, tomografía axial computarizada ${ }^{5}$ o resonancia magnética. El ultrasonido puede mostrar una masa pancreática heterogénea sin septos en su interior. La mejor opción diagnóstica de estos tumores es la tomografía axial computarizada abdominal, en donde se encuentra una masa heterogénea hiperdensa con cápsula definida; en $10 \%$ de los casos, el TSPP contiene líquido en su interior; en 30\% se observan calcificaciones capsulares o periféricas. En la resonancia magnética el hallazgo es de una lesión heterogénea, hiperintensa en 90\% de las veces; la biopsia por aspiración con aguja fina guiada por ultrasonido endoscópico tiene sensibilidad de $81 \%$ y especificidad de $87.5 \%$ para tumores no endocrinos. La tomografía por emisión de positrones no agrega mayor información.
El tratamiento de elección ${ }^{6}$ es quirúrgico; con respecto a su localización, el TSPP se ubica en la cabeza del páncreas en 33 a 40\%; en el cuerpo, 14 a 28\%; y en la cola, 32 a $50 \%$; las opciones quirúrgicas van desde la enucleación simple a la pancreatectomía distal y la duodenopancreatectomía. En todos los casos, se debe intentar preservar la mayor cantidad de tejido pancreático, incrementándose el riesgo de fístula con la enucleación simple. En el caso descrito se realizó pancreatectomía distal con esplenectomía. Se debe tener presente que el tamaño del tumor no debe considerarse predictor de irresecabilidad.

La supervivencia ${ }^{7}$ con tratamiento quirúrgico es de $97 \%$ a cinco años; la recurrencia puede llegar a 10-15\%. En los casos acompañados de lesiones multicéntricas (no presentes en este caso) a partir de tejido pancreático ectópico en hígado, retroperitoneo, mesocolon y epiplón o metástasis sincrónicas, debe intentarse la resección de estas lesiones, ya que el pronóstico continúa siendo satisfactorio, pues la diseminación no se considera un factor predictivo negativo de supervivencia.

La quimioterapia se ha propuesto en los tumores irresecables o con múltiples metástasis a hígado, pero no existe un esquema estandarizado (el caso presentado no fue candidato a terapia adyuvante, ya que el tumor pudo ser resecado en su totalidad, además de no existir lesiones multicéntricas ni metástasis hepáticas).

Macroscópicamente, la existencia de cápsula tumoral y hemorragia intratumoral, así como la inmunohistoquímica del tejido tumoral ${ }^{8}$ permiten distinguirlo de otros tumores del páncreas. Microscópicamente, el tumor presenta áreas sólidas alternando con cambios degenerativos que producen un patrón pseudopapilar ${ }^{9}$ característico de células epiteliales en varias capas alrededor de un tallo central fibrovascular; las áreas sólidas muestran necrosis, macrófagos esponjosos, gránulos de colesterol y calcificaciones. Hasta en $15 \%$ de los casos hay metástasis o recurrencia del tumor. Los sitios más frecuentemente afectados por las metástasis son el hígado (28\%), la vena cava $(27 \%)$ y el bazo (17\%). El comportamiento maligno ${ }^{10}$ puede ser predicho ante evidencia de invasión perineural o a vasos sanguíneos, con o sin invasión profunda a los tejidos circundantes, además de alto grado de pleomorfismo celular e índice mitótico elevado y patrón celular no diferenciado.

\section{CONCLUSIONES}

Se presenta el caso de una persona en quien se llegó al diagnóstico por ultrasonido y tomografía de una masa localizada en el páncreas, que correspondió histológicamente a un tumor sólido pseudopapilar del páncreas. Se trata del sexto reportado en México y el primero en hombres, ya que hasta 2012 se encontraban descritos 335 casos en el género masculino. Las manifestaciones 
clínicas del paciente incluyeron dolor abdominal, sensación de plenitud o saciedad temprana, masa abdominal, náusea y vómito.

La tomografía axial computarizada es el método de gabinete de elección, revelando una masa heterogénea grande encapsulada. El diagnóstico se realiza mediante biopsia y la cirugía tiene un nivel de curación excelente, con supervivencia del $97 \%$ a los cinco años.

\section{REFERENCIAS}

1. Cervantes-Monteil F, Florez-Zorrilla C, Alvarez-Martínez I. Solidcystic pseudopapillary tumor of the pancreas: acute post-traumatic presentation. Case report and review of the literature. Rev Gastroenterol Mex. 2002; 67 (2): 93-96.

2. Camacho-Aguilera JF, Romero-Mejía C, Valenzuela-Espinoza A. Solid pseudopapillary tumor of the pancreas: case report and literature review. Cir Cir. 2010; 78: 73-78.

3. Law KJ, Ahmed A, Vikesh K, Singh KV, Akshintala VS, Olson et al. A systematic review of solid-pseudopapillary neoplasms, are these rare lesions? Pancreas. 2014; 43: 331-337.
4. Gurevich LE, Kazantseva IA, Sokolova IN, Korsakova NA, Kuzovleva EI, Chistiakova OA et al. Solid pseudopapillary tumors of the pancreas: clinical and morphological characteristics, specific features of their immunophenotype, and diagnostic problems. Arkhiv Patol. 2014; 5: 44-54.

5. Lee JH, Byun JH, Kim JH, Lee SS, Kim HJ, Lee MG. Solid pancreatic tumors with unilocular cyst-like appearance on CT: differentiation from unilocular cystic tumors using CT. Korean J Radiol. 2014; 15 (6): 704-711.

6. Shuja A, Alkimawi KA. Solid pseudopapillary tumor: a rare neoplasm of the pancreas. Gastroenterol Rep (Oxf). 2014; 2 (2): 145-149.

7. Jana T, Shroff J, Bhutani MS. Pancreatic cystic neoplasms: review of current knowledge, diagnostic challenges, and management options. J Carcinog. 2015; 14: 3-8.

8. Yagcı A, Yakan S, Coskun A, Erkan N, Yıldırım M, Yalcın E, Postacı $\mathrm{H}$. Diagnosis and treatment of solid pseudopapillary tumor of the pancreas: experience of one single institution from Turkey. W J Surg Oncol. 2013; 11: 308-312.

9. Gurevich L, Kazantseva I, Isakov AV, Korsakova N, Egorov A, Kubishkin V et al. The analysis of immunophenotype of gastrin-producing tumors of the pancreas and gastrointestinal tract. Cancer. 2003; 98: 1967-1976.

10. Yu PF, Hu ZH, Wang XB et al. Solid pseudopapillary tumor of the pancreas: a review of 553 cases in Chinese literature. World J Gastroenterol. 2010; 16: 1209-1214. 